\title{
A TIGHT BOUND ON THE LENGTH OF ODD CYCLES IN THE INCOMPATIBILITY GRAPH OF A NON-C1P MATRIX
}

\author{
MEHRNOUSH MALEKESMAEILI, CEDRIC CHAUVE, AND TAMON STEPHEN
}

\begin{abstract}
A binary matrix has the consecutive ones property $(\mathrm{C} 1 \mathrm{P})$ if it is possible to order the columns so that all 1s are consecutive in every row. In [McConnell, SODA 2004 768-777] the notion of incompatibility graph of a binary matrix was introduced and it was shown that odd cycles of this graph provide a certificate that a matrix does not have the consecutive ones property. A bound of $k+2$ was claimed for the smallest odd cycle of a non-C1P matrix with $k$ columns. In this note we show that this result can be obtained simply and directly via Tucker patterns, and that the correct bound is $k+2$ when $k$ is even, but $k+3$ when $k$ is odd.
\end{abstract}

\section{INTRODUCTION}

A binary matrix has the Consecutive Ones Property (C1P), if there exists a permutation of its columns that makes the 1s consecutive in every row. It was first introduced by Fulkerson and Gross in [FG65] as special case of deciding whether a graph is an interval graph, and has important applications in computational biology, see for example [AKWZ94]. The problem of deciding whether a given binary matrix has the C1P can be solved efficiently [BL76]. Clearly, checking the claim that a matrix is C1P is easy, provided a valid permutation of the columns is given. However it is not obvious how to certify that a matrix is not C1P.

The first structural result on non-C1P matrices is due to Tucker, who proved in Tuc72] that a binary matrix is not $\mathrm{C} 1 \mathrm{P}$ if and only if it contains a submatrix from one of five families of binary matrices known as Tucker patterns, that define then a natural family of certificates for non-C1P matrices. Tucker patterns can be detected in polynomial time DGN10.

McConnell, in [McC04, defined a simple and elegant certificate for non-C1P matrices. He introduced the notion of the incompatibility graph of a binary matrix, and proved that a matrix is $\mathrm{C} 1 \mathrm{P}$ if and only if this graph is bipartite. Hence, an odd cycle in this graph is a non-C1P certificate. He claimed that the incompatibility graph of a non-C1P matrix with $k$ columns always has an odd cycle of length at most $k+2$ and proposed a linear time algorithm to compute such a cycle, more efficient than the currently best algorithms to detect Tucker patterns [DGN10].

In this note we correct the bound McConnell gave in [McC04] for the length of the odd cycle of the incompatibility graph. We prove that the incompatibility graph of a non-C1P matrix with $k \geq 4$ columns has the smallest odd cycle of length at most $k+2$ if $k$ is odd 


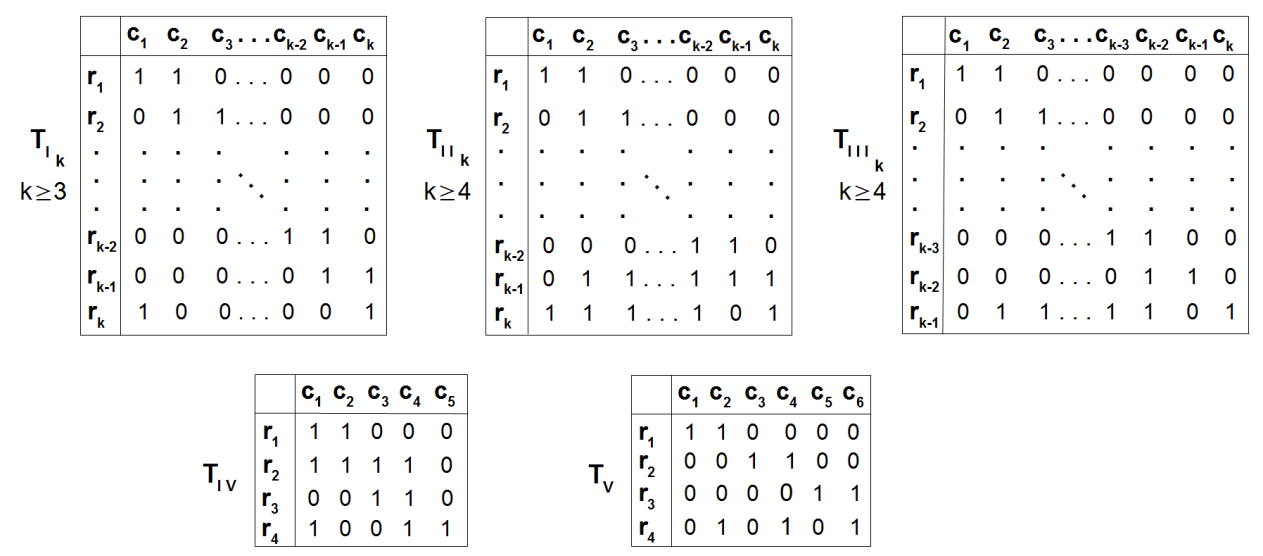

Figure 1. The five Tucker patterns

and $k+3$ if $k$ is even, and that this bound is tight. Our approach relies on investigating the odd cycles of the incompatibility graphs of Tucker patterns.

\section{Preliminaries}

2.1. Tucker patterns. Tucker characterized C1P matrices via minors known as "Tucker patterns" illustrated in Figure 1.

Lemma 2.1. Tuc72 A binary matrix has the Consecutive Ones Property if and only if it contains none of the five Tucker patterns as a submatrix.

These patterns are the minimal structures that obstruct the matrix $M$ from having the $\mathrm{C} 1 \mathrm{P}$, i.e. removing a row from these structures result in a C1P-matrix. In this present note, for all of the five Tucker matrices we consider the order of columns given in Figure 1.

2.2. Incompatibility graph. The concepts of incompatibility and forcing graphs were introduced by McConnell in McC04. Let $M$ be an $m \times n$ binary matrix with rows $R=$ $\left\{r_{1}, r_{2}, \ldots, r_{m}\right\}$ and columns $C=\left\{c_{1}, c_{2}, \ldots, c_{n}\right\}$. The incompatibility graph of $M$ is an undirected graph $G_{M}=(V, E)$, whose vertices are pairs $\left(c_{i}, c_{j}\right)$ (for $i, j=1, \ldots, n, i \neq j$ ). Two vertices $\left(c_{i}, c_{j}\right)$ and $\left(c_{j}, c_{k}\right)$ are adjacent, if one of the following holds:

(1) $c_{i}=c_{k}$.

(2) There exists a row $r_{l}$ in $M$ such that $M_{l i}, M_{l k}=1$ but $M_{l j}=0$.

We sometimes refer to these edges as type 1 or type 2 , as appropriate. These edges represent incompatible pairs of orderings, i.e. each edge corresponds to two relative orderings of the columns that cannot appear simultaneously in a consecutive ones ordering of the matrix. McConnell noted that the incompatibility graph is bipartite if and only if the matrix is C1P. Thus odd cycles in the incompatibility graph certify that a matrix is not C1P.

The forcing graph $F_{M}=\left(V, E^{\prime}\right)$ is an undirected graph whose vertex set is same as that of $G_{M}$ and whose edge set is a set of all pairs $\left(\left(c_{i}, c_{j}\right),\left(c_{k}, c_{j}\right)\right)$ where $\left(\left(c_{i}, c_{j}\right),\left(c_{j}, c_{k}\right)\right)$ is an 
edge of $G_{M}$. It is not hard to see that the incompatibility graph and the forcing graph both have $n(n-1)$ vertices and are symmetric. As with the incompatibility graph, the forcing graph can be used to certify that a matrix is not C1P: a path in this graph from $\left(c_{i}, c_{j}\right)$ to $\left(c_{j}, c_{i}\right)$ for and $i, j$, represents a chain of implications ("forcings") leading to a contradiction.

In fact, McConnell observed that these certificates are almost the same: such a path in the forcing graph can be transformed to a cycle in the incompatibility graph and vice versa.

Lemma 2.2. If there exists a path with $m$ vertices in $F_{M}$ between $\left(c_{i}, c_{j}\right)$ and $\left(c_{j}, c_{i}\right)$ there is an odd cycle of length $m-1$ (if $m$ is even) or $m$ (if $m$ is odd) in $G_{M}$ containing the vertex $\left(c_{i}, c_{j}\right)$. Conversely, if there is an odd cycle of length $m$ in $G_{M}$ containing $\left(c_{i}, c_{j}\right)$ there is a path with at most $m+1$ vertices in $F_{M}$ from $\left(c_{i}, c_{j}\right)$ to $\left(c_{j}, c_{i}\right)$.

Proof. Without loss of generality the path in $F_{M}$ is:

$$
P: \quad\left(v_{1}=\left(c_{i}, c_{j}\right), v_{2}, v_{3}, v_{4}, \ldots, v_{m}=\left(c_{j}, c_{i}\right)\right)
$$

Let $v_{k}^{\prime}=\left(c^{\prime}, c\right)$ when $v_{k}=\left(c, c^{\prime}\right)$. Then in $G_{M}$ we can build the walk:

$$
P^{\prime}: \quad\left(v_{1}, v_{2}^{\prime}, v_{3}, v_{4}^{\prime}, \ldots, v_{m}^{\left({ }^{\prime}\right)}\right)
$$

When $m$ is even, the final vertex in this walk is $v_{m}^{\prime}=v_{1}$, and we have a cycle with $m-1$ vertices. When $m$ is odd, the final term is $v_{m}$, and we can complete the cycle using the type 1 edge $\left(v_{m}, v_{m}^{\prime}=v_{1}\right)$. In this case the odd cycle has length $m$.

Similarly, an odd cycle of length $m$ in $G_{M}$ can be transformed into a walk of length $m+1$ in $F_{M}$ by performing the reverse operation on the walk with even length $m+1$ by taking the cycle vertices starting an ending in $v_{1}$. Note that if this path contains some type 1 edge $\left(v_{k}, v_{k}^{\prime}\right)$ in $G_{M}$, this becomes a trivial edge $\left(v_{k}, v_{k}\right)$ or $\left(v_{k}^{\prime}, v_{k}^{\prime}\right)$ in $F_{M}$ and should be contracted, reducing the length of the found path.

Given $G_{M}\left(F_{M}\right)$, we define $G_{M}^{1}\left(F_{M}^{1}\right)$ and $G_{M}^{2}\left(F_{M}^{2}\right)$ to be the subgraphs induced by the vertex sets $V_{1}=\left\{\left(c_{i}, c_{j}\right) \mid i<j\right\}$ and $V_{2}=\left\{\left(c_{i}, c_{j}\right) \mid i>j\right\}$ respectively. We observe that the two pairs of subgraphs are isomorphic.

Suppose now that we build $G_{M}$ and $F_{M}$ graph for a given Tucker pattern $M$ from Figure 1 by first generating the type 1 edges of $G_{M}$ and then adding the edges generated by each row in turn, beginning at the top. Edges $e=\left(\left(c_{i}, c_{j}\right),\left(c_{l}, c_{j}\right)\right)$ and $e^{\prime}=\left(\left(c_{j}, c_{i}\right),\left(c_{j}, c_{l}\right)\right)$ in $F_{M}$ are generated by triples $(i, j, l)$ from a given row $r$ exactly when $M_{r i}, M_{r l}=1$ but $M_{r j}=0$. The edges corresponding to the rows from the top of the matrix then come in pairs $e, e^{\prime}$, where one is contained in $F_{M}^{1}$ and the other in $F_{M}^{2}$. As we descend the rows, the ones in the rows are consecutive until we reach the final row, $r_{t}$, that has gaps between its 1 s entries.

The edges of $F_{M}$ generated by the gaps in $r_{t}$, i.e. triples $(i, j, l)$ of columns where $i<j<l$ are the only edges which go between $V_{1}$ and $V_{2}$. We call these edges critical. 


\section{Finding ODD CyCles USing Tucker CONFigurations}

We now give a tight bound on the smallest odd cycle in the incompatibility graph of a non-C1P matrix using Tucker matrices.

Theorem 3.1. The length of the smallest odd cycle in the incompatibility graph of a binary matrix with $k \geq 4$ columns is at most $k+2$ if $k$ is odd or $k+3$ is $k$ is even, and this bound is tight.

We begin by remarking that since any non-C1P matrix $M$ contains a Tucker pattern as a submatrix, we can restrict our attention to Tucker patterns when looking for short odd-cycles in the incompatibility graph. This is because if we look at the subgraph of the incompatibility graph induced by considering only the columns (vertices) and rows (edges) of $M\left(G_{M}\right)$ containing the Tucker pattern, we get exactly the incompatibility graph of the submatrix, which has at most as many columns as $M$. So the upper bound for Tucker patterns holds for all $M$, and the worst case for a given number of columns will occur at a Tucker pattern.

We remark that for $k \leq 2$ all binary matrices have the $\mathrm{C} 1 \mathrm{P}$, and for $k=3$ if a matrix is not $\mathrm{C} 1 \mathrm{P}$ it must contain the Tucker pattern $T_{I_{3}}$ as a submatrix, and thus have an odd cycle of length 3 in its incompatibility graph, see Section 3.1. For $k \geq 4$, the tight bound of $k+2$ or $k+3$ is attained by $T_{I I I_{k}}$, see Section 3.3 . We proceed to analyze each Tucker pattern separately.

3.1. First Tucker pattern. $T_{I_{k}}$ is shown in Figure 1 ; it is a square matrix of size $k$ where $k \geq 3$.

Lemma 3.2. For $k \geq 3$, the length of the smallest odd cycle in the incompatibility graph of $T_{I_{k}}$ is $k$ when $k$ is odd and $k+1$ when $k$ is even.

Proof. We find a path in $F_{M_{1_{k}}}$ in $T_{I_{k}}$ between $\left(c_{1}, c_{k-1}\right)$ and $\left(c_{k-1}, c_{1}\right)$. Since $M_{11}, M_{12}=$ 1 but $M_{1 k-1}=0$, we have that $\left(\left(c_{1}, c_{k-1}\right),\left(c_{2}, c_{k-1}\right)\right)$ is an edge of $F_{M_{I_{k}}}$. Similarly $\left(\left(c_{i}, c_{k-1}\right),\left(c_{i+1}, c_{k-1}\right)\right)$ is an edge of $F_{M_{I_{k}}}$ for $i=2, \ldots, k-3$ using row $i$ of $M$. Using row $k-2$, we get that $\left(c_{k-2}, c_{k-1}\right)$ forces $\left(c_{k-2}, c_{k}\right)$ and using row $k-1$ that $\left(c_{k-2}, c_{k}\right)$ forces $\left(c_{k-1}, c_{k}\right)$. Observe that $e=\left(\left(c_{1}, c_{k-1}\right),\left(c_{k}, c_{k-1}\right)\right)$ is a critical edge of $F_{M_{1_{k}}}$. Therefore $\left(c_{1}, c_{k-1}\right),\left(c_{2}, c_{k-1}\right),\left(c_{3}, c_{k-1}\right), \ldots,\left(c_{k-2}, c_{k-1}\right),\left(c_{k-2}, c_{k}\right),\left(c_{k-1}, c_{k}\right),\left(c_{k-1}, c_{1}\right)$ is a path with $k+1$ vertices in $F_{M_{I_{k}}}$. By Lemma 2.2 , this gives the required cycle.

Finally, we note that if there is any odd cycle in the incompatibility graph of length less than $k$, we would derive a contradiction to the C1P using fewer than $k$ columns, contradicting the minimality of the Tucker pattern. Thus the length of this odd cycle is in fact minimal.

3.2. Second Tucker pattern. For $k \geq 4, T_{I I_{k}}$ is a square matrix of size $k$. We use a strategy to find a cycle in the incompatibility graph of $T_{I I_{k}}$ that is similar to that of $T_{I_{k}}$.

Lemma 3.3. The smallest odd cycle in the incompatibility graph of $T_{I I_{k}}$ has length $k$ when $k$ is odd and $k+1$ when $k$ is even. 


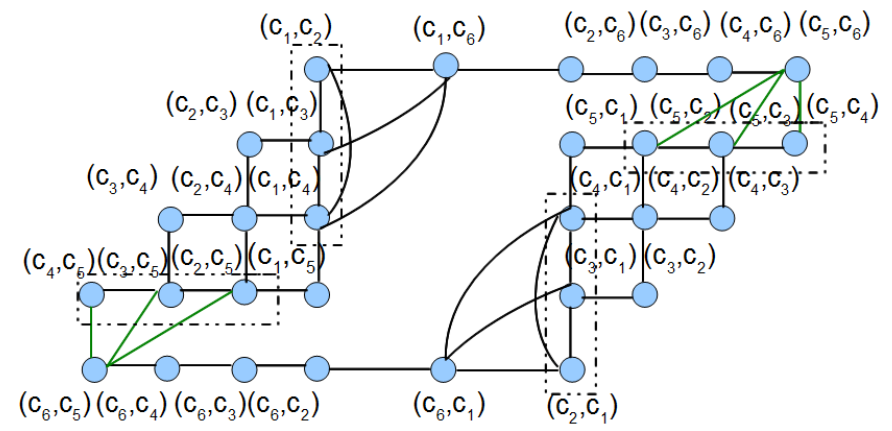

FIGURE 2. $F_{M_{I I_{6}}}$

Proof. From row $i$ of the matrix for $i=1, \ldots, k-2$, we get that $\left(c_{i}, c_{k}\right)$ forces $\left(c_{i+1}, c_{k}\right)$. From row $k-1,\left(c_{1}, c_{k}\right)$ forces $\left(c_{1}, c_{k-1}\right)$. Finally, $\left(\left(c_{1}, c_{k-1}\right),\left(c_{k}, c_{k-1}\right)\right)$ is a critical edge. Then $\left(c_{1}, c_{k}\right),\left(c_{2}, c_{k}\right), \ldots,\left(c_{k-1}, c_{k}\right),\left(c_{k-1}, c_{1}\right),\left(c_{k}, c_{1}\right)$ is a path of length $k+1$ in the forcing graph of $T_{I I_{k}}$. Using Lemma 2.2 we can find an odd cycle of length either $k$ or $k+1$ containing all rows of the pattern. Again the minimality of the Tucker pattern ensures that we cannot have a cycle of length less than $k$.

3.3. Third Tucker pattern. Now we consider the third Tucker pattern that has $(k-1)$ rows and $k$ columns where $k \geq 4$.

Lemma 3.4. The smallest odd cycle in the incompatibility graph of the third Tucker pattern has length $k+2$ if $k$ is odd and $k+3$ if $k$ is even.

Proof. In this case, because we need to prove a non-trivial lower bound, we will describe the full structure of $F_{M_{I I I}}$. The graph $F_{M_{I I I_{6}}}$ is illustrated in Figure 2 and captures the features we are interested in. Consider first the portion of the graph generated by excluding the last row and column on $M_{I I I_{k}}$. In this case each row has a unique pair of 1 entries; these can be combined with any zero entry to get a forcing triple. The result is a triangular grid on $V_{1}$ (and symmetrically, $V_{2}$ ), where vertex $\left(c_{i}, c_{j}\right)$ is connected to all of $\left(c_{i \pm 1}, c_{j}\right)$ and $\left(c_{i}, c_{j \pm 1}\right)$ that are also vertices of $V_{1}$ with coordinates between 1 and $k-1$ and in increasing order. Now, returning our attention the last column, we see it generates a path $\left(c_{1}, c_{k}\right),\left(c_{2}, c_{k}\right), \ldots\left(c_{k-1}, c_{k}\right)$ by considering the pair of ones in each row in turn.. These two components, and their symmetric copies in $V_{2}$ are the entirety of the graph if we exclude the final row.

The first zero from the final row combines with the many pairs of ones to connect the two components of $V_{1}$ by fusing $\left(c_{1}, c_{2}\right),\left(c_{1}, c_{3}\right), \ldots\left(c_{1}, c_{k-2}\right)$ and $\left(c_{1}, c_{k}\right)$ (but not $\left.\left(c_{1}, c_{k-1}\right)\right)$ into a clique. Finally the second zero (in row $k-1)$ produces the critical edges $\left(c_{i}, c_{k}\right),\left(c_{k}, c_{k-1}\right)$ for $i=2, \ldots, k-2$ and fuses all these vertices into a clique.

We can then see that a path with $(k+3)$ vertices in $F_{M_{I I I}}$ from $\left(c_{1}, c_{k}\right)$ to $\left(c_{k}, c_{1}\right)$ is given by: $\left(c_{1}, c_{k}\right),\left(c_{2}, c_{k}\right), \ldots,\left(c_{k-1}, c_{k}\right),\left(c_{k-1}, c_{2}\right),\left(c_{k-1}, c_{1}\right),\left(c_{k-2}, c_{1}\right),\left(c_{k}, c_{1}\right)$. By Lemma 2.2, this 


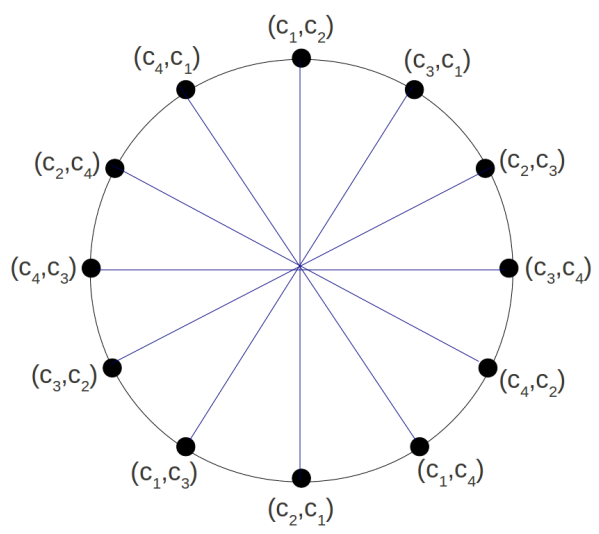

FIGURE 3. Incompatibility Graph of $T_{I I I_{4}}$

gives the required cycle in the incompatibility graph of length $(k+3)$ if $k$ is even, and $(k+2)$ if $k$ is odd.

To prove that this is shortest, it suffices to show that this is the shortest path between $\left(c_{i}, c_{j}\right)$ and $\left(c_{j}, c_{i}\right)$ for some $i, j$ in $F_{M_{I I I_{k}}}$, since if there is a shorter odd cycle in the incompatibility graph of length $(k+1)$ with $k$ even, or $k$ with $k$ odd, by Lemma 2.2 there would be a path of length at most $k+2$ between some $\left(c_{i}, c_{j}\right)$ and $\left(c_{j}, c_{i}\right)$.

We can see that there is no shorter path by contracting the groups of vertices illustrated in Figure 2, i.e. $\left(c_{1}, c_{j}\right)$ for $j=2,3, \ldots k-2 ;\left(c_{i}, c_{k-1}\right)$ for $i=2,3, \ldots k-2$; and the symmetric groups on $F_{V_{2}}$. This will not increase the distance between any pair of vertices. We can see that what remains is $2 k-2$ cycle with vertices $\left(c_{i}, c_{j}\right)$ opposite $\left(c_{j}, c_{i}\right)$, an additional part of $F_{V_{1}}$ attached to the two contracted vertices, and symmetrically in $F_{V_{2}}$. The shortest path between opposite vertices on the cycle has $(k+3)$ vertices, as does the shortest path between any of additional vertices in $F_{V_{1}}$ and those of $F_{V_{2}}$, though for some choices of $(i, j)$ the shortest path from $\left(c_{i}, c_{j}\right)$ to $\left(c_{j}, c_{i}\right)$ may be longer.

Taking $k \geq 4$ even, this gives a family of counter examples to Theorem 6.1 of McConnell in [McC04. For example, taking $k=4$, we have $M=\left(\begin{array}{llll}1 & 1 & 0 & 0 \\ 0 & 1 & 1 & 0 \\ 0 & 1 & 0 & 1\end{array}\right)$. Then $F_{M}$ is a 12-cycle and $G_{M}$ is a 12-cycle with 6 chords added between opposite vertices of the cycle. It is clear that the smallest odd cycle is of length 7 . This graph is shown in Figure 3.

3.4. Fourth Tucker pattern. The fourth Tucker pattern is of size 4 by 5 , with $\left(\left(c_{1}, c_{3}\right)\right.$, $\left.\left(c_{5}, c_{3}\right)\right)$ as a critical edge of $F_{M_{I V}}$. Here $\left(c_{1}, c_{3}\right)$ forces $\left(c_{5}, c_{3}\right),\left(c_{5}, c_{3}\right)$ forces $\left(c_{5}, c_{2}\right),\left(c_{5}, c_{2}\right)$ forces $\left(c_{4}, c_{2}\right),\left(c_{4}, c_{2}\right)$ forces $\left(c_{3}, c_{2}\right)$ and $\left(c_{3}, c_{2}\right)$ forces $\left(c_{3}, c_{1}\right)$, which gives a path with 6 vertices in $F_{M_{I V}}$ and an odd cycle of length 5 in the incompatibility graph of $T_{I V}$.

3.5. Fifth Tucker pattern. The fifth Tucker pattern shown in Figure 1 is 4 by 6 . It can be observed that $\left(\left(c_{2}, c_{3}\right),\left(c_{6}, c_{3}\right)\right)$ is a critical edge of $F_{M_{V}}$. Now $\left(c_{2}, c_{3}\right)$ forces $\left(c_{6}, c_{3}\right)$ which forces $\left(c_{6}, c_{4}\right) ;\left(c_{6}, c_{4}\right)$ forces $\left(c_{5}, c_{4}\right),\left(c_{5}, c_{4}\right)$ forces $\left(c_{5}, c_{2}\right),\left(c_{5}, c_{2}\right)$ forces $\left(c_{5}, c_{1}\right)$ which forces $\left(c_{6}, c_{1}\right)$. $\left(c_{6}, c_{1}\right)$ forces $\left(c_{4}, c_{1}\right)$ which forces $\left(c_{4}, c_{2}\right) ;\left(c_{4}, c_{2}\right)$ forces $\left(c_{3}, c_{2}\right)$. This gives a path 
with 10 vertices in $F_{M_{V}}$ and an odd cycle of length 9 in $T_{V}$. In this case the length of the smallest odd cycle also attains the bound of Theorem 3.1 .

Combining these five case allows us to conclude Theorem 3.1 .

Remark 1. Running the partition refinement algorithm of [McC04] on $M_{I I I_{k}}$ may generate an odd cycle of length as much as $2 k-1$ for the certificate, depending on which critical edge is processed from the last row.

\section{Acknowledgments}

All three authors were supported by NSERC Discovery Grants. The authors thank Ross McConnell for helpful discussion.

\section{REFERENCES}

[AKWZ94] F. Alizadeh, R. M. Karp, D. K. Weisser, and G. Zweig, Physical mapping of chromosomes using unique probes, Proceedings of the fifth annual ACM-SIAM symposium on Discrete algorithms (Philadelphia, PA, USA), SODA'94, Society for Industrial and Applied Mathematics, 1994, pp. 489-500.

[BL76] K. S. Booth and G. S. Lueker, Testing for the consecutive ones property, interval graphs, and graph planarity using PQ-tree algorithms, J. Comput. Syst. Sci. 13 (1976), 335-379.

[DGN10] M. Dom, J. Guo, and R. Niedermeier, Approximation and fixed-parameter algorithms for consecutive ones submatrix problems, J. Comput. Syst. Sci. 76 (2010), 204-221.

[FG65] D. R. Fulkerson and O. A. Gross, Incidence Matrices and Interval Graphs, Pacific J. Math. 15 (1965), 835-855.

[McC04] R. M. McConnell, A certifying algorithm for the consecutive-ones property, Proceedings of the fifteenth annual ACM-SIAM symposium on Discrete algorithms (Philadelphia, PA, USA), SODA'04, Society for Industrial and Applied Mathematics, 2004, pp. 768-777.

[Tuc72] A. Tucker, A structure theorem for the consecutive 1's property, J. Combinat. Theory, Series B 12 (1972), no. 2, 153-162.

Department of Mathematics, Simon Fraser University, Burnaby, BC, Canada

E-mail address: \{mmalekes, cedric.chauve, tamon\}@sfu.ca 